\title{
Stretching and heating single DNA molecules with optically trapped gold- silica Janus particles
}

Sabrina Simoncelli ${ }^{1+*}$, Samuel Johnson ${ }^{1 *}$, Franziska Kriegel ${ }^{2}$, Jan Lipfert ${ }^{2,3 *}$ and Jochen

Feldmann $^{1,3}$

${ }^{1}$ Photonics and Optoelectronics Group, Department of Physics and Center for Nanoscience

(CeNS), Ludwig-Maximilians-Universität München, Amalienstraße 54, Munich, 80799, Germany

${ }^{2}$ Department of Physics and Center for Nanoscience (CeNS), Ludwig-Maximilians-Universität München, Geschwister-Scholl-Platz 1, Munich, 80539, Germany

${ }^{3}$ Nanosystems Initiative Munich (NIM), Schellingstraße 4, 80539 Munich, Germany

These authors contributed equally to this work

†Present address: The Blackett Laboratory, Department of Physics, Imperial College, London SW7 2AZ, UK.

Abstract: Self-propelled micro and nano-scale motors are capable of autonomous motion typically by inducing local concentration gradients or thermal gradients in their surrounding medium. This is a result of the heterogeneous surface of the self-propelled structures that consist of materials with different chemical or physical properties. Here we present a selfthermophoretically driven Au-silica Janus particle that can simultaneously stretch and partially melt a single double-stranded DNA molecule. We show that the effective force acting on the DNA molecule is in the $\sim \mathrm{pN}$-range, well suited to probe the entropic stretching regime of DNA, and we demonstrate that the local temperature enhancement around the gold side of the particle produces partial DNA de-hybridization. 
Keywords: self-thermophoresis, Janus particles, optical trapping, DNA stretching, DNA dehybridization, $p N$ forces.

Self-propelled nano- and micrometer structures, capable of converting energy into directed motion, have shown great potential in a broad range of applications ranging from biomedicine to environmental remediation. ${ }^{1,2}$ Specifically, self-powered micro-motors have already proven capable of delivering cargo molecules to desired locations, ${ }^{3}$ drilling a hole into soft tissues ${ }^{4}$ and even transporting themselves towards fixed cancer cells to subsequently kill them. ${ }^{5}$ The accurate motion control of these self-propelled colloids often depends on their anisotropic response to their chemical environment, creating a local gradient or field that drives their motion. Asymmetric particles that present two sides of different materials in their composition are typically called Janus particles. While some applications require fully autonomous navigation mechanisms so that the Janus particles can reach specific target locations, i.e. control of rotational and translational diffusion, others use external magnetic or electric fields to control the orientation and propulsion direction of the particles. For example, using a highly defocused and weak illumination beam in combination with feedback-loops, it is possible to control the active Brownian motion of metal-dielectric Janus particles both in space and time. ${ }^{6,7}$ Essentially, the metal-coated hemisphere of the Janus particle absorbs light and heats up more efficiently than the other hemisphere, which results in a local temperature gradient that drives the particle motion by self-thermophoresis. On the other hand, it is also possible to combine thermophoretic forces with optical forces to control the motion of Janus particles. ${ }^{8}$ Our group has recently shown the ability to control the self-propelled motion of silica micron-sized particles half-functionalized with a thin layer of gold using a highly focused laser. ${ }^{9,10}$ In our 
configuration, the axial propulsion mechanism of the Janus particle in the optical trap is achieved by self-thermophoresis, while the optical forces acting on the particle determine the rotational orientation and directionality of the motion of the particle inside the optical trap. Hence, thermophoretic forces only control the degree of the axial motion. In fact, we demonstrated that the axial position of the Janus particle in the optical trap is not fixed, but it depends on the laser power, and as such we envision that it can be used as a highly sensitive force transducer with an additional heating capability.

Optical tweezers have become a powerful tool for manipulating a variety of micro- and nanoscale objects, such as dielectric particles, ${ }^{11}$ gold nanoparticles ${ }^{12-15}$ and cells, ${ }^{16}$ providing unprecedented insights into the structure, mechanics, conformational transitions, and function of biological macromolecules. ${ }^{17-20}$ Several variations to the standard optical tweezers technique have been used to study the mechanical properties of single DNA molecules, including the tethered assay, ${ }^{21}$ the two-bead assay, ${ }^{22}$ angular optical trapping, ${ }^{23}$ and active position-clamping. ${ }^{24}$ In fact, optical tweezers have also proven useful to study DNA hybridization kinetics when combine with plasmonic heating in an optical trap. ${ }^{25}$ In these configurations, the DNA molecule is usually stretched by laterally moving the trapped particles with respect to the axis of the optical trap. The versatility and precision afforded by these lateral displacement can, however, be sometimes accompanied by geometrical artifacts, i.e. rotation instead of lateral displacement when the DNA molecule is pulled under oblique angles. Therefore, alternative approaches to axially pull single molecules inside an optical trap can become useful to minimize these effects when studying short DNA molecules. Indeed, while many of the standard manipulation techniques, notable atomic force microscopy. optical and magnetic tweezers, ${ }^{26,27}$ have been developed into mature and commercially available instruments, there still is a strong need for 
alternative strategies to enhance and broaden the toolbox of single-molecule techniques. Examples of recently developed approaches to manipulating individual molecules include centrifugal force application, ${ }^{28}$ acoustic force spectroscopy, ${ }^{29}$ optical "pushing", ${ }^{30}$ and a "DNA pulley". ${ }^{31}$ The ability to achieve continuous and directed motion on the nano- to micrometer scale through externally controlled electric, magnetic or acoustic fields could open up a range of applications to single-molecule manipulation in biological systems. Here, we use active plasmonic self-propelling micron-sized particles to stretch and also simultaneously heat individual macromolecules. As a proof-of-concept, we experimentally prove this approach on double-stranded DNA (dsDNA) molecules, whose stretching response has been described in detail in a large number of publications and which provides a well characterized test system. ${ }^{17,32}$

\section{Results and Discussion}

Figure 1 illustrates the principle of the self-propelled motion of our gold-silica micronsized Janus particles, which are synthesized by thermally evaporating a thin layer of gold over a monolayer of $1.3 \mu \mathrm{m}$ silica spheres (Methods and Figure 1a). ${ }^{9,10} \mathrm{Au}$-silica Janus particles are optically trapped with an IR laser beam that also acts as the energy source for the heat generation (Figure 1c, left). While heat generation by water absorption of $1064 \mathrm{~nm}$ laser light is negligible $(\sim 0.2 \mathrm{~K})$ in the range of trapping laser powers used in our experiments $(<20 \mathrm{~mW}),{ }^{33}$ the gold cap of the particle efficiently absorbs the light of the trapping laser (Fig. S1) increasing the surface temperature of the gold hemisphere. The symmetry breaking induced by the temperature gradient between the gold and silica hemispheres of the particle allows it to self-propel inside the optical trap. Particularly, Janus particles self-propel against the direction of the beam propagation. The directionality of the motion is a result of the orientation of the particle in the optical trap, with the gold layer facing in the direction of the beam propagation. The orientation 
of the particle is determined by the scattering force, which is stronger in the gold-coated hemisphere and pushes the particle towards the direction of the beam. To counteract the thermal gradient, the Janus particle moves in the opposite direction from the gold hemisphere. Ultimately, the particle reaches a stable trapping position above the optical focus, that is determined by the balance of the thermophoretic and the optical forces acting on it (Figure 1c, left).

We stretch single dsDNA molecules with self-thermophoretically driven micron-sized gold-silica Janus particles (Figure 1). The Janus particle, which is optically trapped and tethered to a $3 \mu \mathrm{m}$-long dsDNA molecule (Figure 1b), acts as handle for exerting controlled forces on the DNA. The dsDNA molecule is attached with one end to the surface of a glass substrate, via a digoxigenin-antidigoxigenin (DIG-antiDIG) linkage, and with the other end to the gold hemisphere of the particle, via a thiol bond (Figure 1c, right, Methods and S2). To optically trap and record the motion of the DNA tethered Janus particle we employed a custom dark-field microscope set-up coupled with a $1064 \mathrm{~nm} \mathrm{CW}$ laser (Methods). We differentiate tethered and untethered Janus particles by observing their Brownian motion. Once a tethered particle is identified, we optically trapped it and gradually increased the power of the trapping beam to study the response of the bound DNA molecule. We recorded videos of the white light scattering pattern of the Janus particle to determine the height of the particle in the optical trap and hence the length of the DNA extension using an in-house MATLAB routine. ${ }^{10}$

The elevation of the DNA-tethered Au-silica Janus particle above the focus of the trapping beam is a result of the balance between the trapping laser power and the DNA stretching response (Figure 1c, right). Figure 2a displays the power dependent height, $h$, of a free (grey squares) and a DNA-tethered (red circles) Au-silica Janus particle with respect to the surface of 
the glass substrate. Free Janus particles elevate as a function of the trapping laser power as previously studied by our group. ${ }^{9,10}$ In the low laser power regime, between 3 to $5 \mathrm{~mW}$, the height of the free Janus particle increases in a nearly linear fashion with the laser power. This behavior is a result of the forces acting on the system: optical, thermophoretic and sedimentation forces (see S3). However, at a critical height value of $\sim 5 \mu \mathrm{m}$ the Janus particle experiences an abrupt jump (Figure 2a). The jump of the Janus particle in the optical trap has also been previously characterized and it is the result of approaching a secondary stable trap positioned above the focus of the laser beam. ${ }^{9}$ Once the Janus particle reaches this secondary trap, it elevates smoothly with the laser power in a similar fashion to the behavior observed before the jump. In contrast, the height power dependency of the DNA-tethered Janus particle displays a markedly different behavior (Figure 2a). The height of the DNA-tethered Janus particle increases approximately linearly with the laser power until reaching a plateau at a height of $\sim 3 \mu \mathrm{m}$. This level height is consistent with the known stretching response of $\mathrm{DNA}^{17,34}$ and the contour length of our DNA construct of $3 \mu \mathrm{m}$ (Figure 1b). Even after the power of $\sim 5 \mathrm{~mW}$ is surpassed (power at which the free Janus particle jumps) the tethered particle remains near the primary trap anchored to the glass substrate with an almost completely extended DNA tether (Figure 2a, green and red highlights). For higher laser powers, > $10 \mathrm{~mW}$, the DNA tether eventually ruptures and the Janus particle elevates to the secondary trap consistent with the behavior of the free particle (Figure 2a, yellow highlight). While the data shown in Figure 2a corresponds to a representative measurement of a single DNA-tethered and a single free Janus particle, we consistently observed the same height-laser power response in repeated measurements on different particles (Fig. S3).

To provide more physical insight into the stretching behaviour of a dsDNA molecule tethered to a self-propelled Janus particle, we translate our set of experimental data (Figure 2a) 
into force-extension curves by estimating the force exerted by the Janus particle to the DNA molecule (i.e. thermophoretic and optical forces, Figure 1c, right). The axial optical forces acting on free and/or DNA-tethered Janus particles, $F_{\text {op }}$, are proportional to the axial displacement of the particle above the focus of the trapping beam, $\Delta h$,

$$
F_{o p}=-k_{z} \Delta h
$$

where $k_{\mathrm{z}}$ is the axial trap stiffness and it is a function of $\Delta h$ and the power of the trapping beam. Similar to the optical tweezers configurations, ${ }^{35}$ the axial trap stiffness can be determined by the axial thermal fluctuations of the particle in the optical trap. In our experimental conditions, we found that the measurement of the transverse displacement of optically trapped Janus particles is much more precise than the measurement of their axial displacement. Specifically, we estimate an error of $\sim 10 \%$ for the accuracy of the axial position versus an error of $\sim 3 \%$ for the accuracy of the transverse ones. Therefore, we assessed the axial trap stiffness of free and DNA-tethered Janus particles, $k_{\mathrm{z}}$, by experimentally establishing the radial trap stiffnes, $k_{\mathrm{r}}$, (see $\mathrm{S} 5$ for a detailed derivation),

$$
k_{z} \approx f\left(R, \omega_{0}, \lambda\right) \cdot k_{r}
$$

where $f$ can be determined using the experimental values of the radius of the particle, $\mathrm{R}$, the trapping wavelength, $\lambda$, and the beam waist in the focal plane, $\omega_{0}$. For a well-aligned optical tweezers system, the trap is radially symmetric, and the radial spring constant is associated with the time-averaged potential energy in the $x$ - and $y$-dimension,

$$
k_{r}=\frac{\delta x^{2}+\delta y^{2}}{\delta x^{2} \delta y^{2}} k_{B} T
$$


where $\delta x$ and $\delta y$ are the deviations in the $x$ and $y$ directions respectively, $k_{\mathrm{B}}$ is Boltzmann's constant and $T$ is the absolute temperature. As an example, Figure 3a displays typical $x$ - and $y$ temporal traces of a free optically trapped Janus particle. The similarity between the two traces reflects the radial symmetry of our system. Finally, by combining Equation (1), (2) and (3) we experimentally determine the optical forces acting on free and/or DNA-tethered Janus particles,

$$
F_{o p}=-\frac{\delta x^{2}+\delta y^{2}}{\delta x^{2} \delta y^{2}} f k_{B} T \Delta h
$$

where $\Delta h$ corresponds to the axial displacement of either free, $\Delta h_{\text {free }}$ or DNA-tethered particles, $\Delta h_{\mathrm{DNA}}$, from the focal plane of the trapping beam. To ascertain the thermophoretic forces acting on optically trapped free and DNA-tethered Janus particles we consider the balancing forces on both systems (Figure 1c). Stable trapping of free Janus particles is achieved when the optical forces acting on the particle are equal and opposite to the thermophoretic force. Hence, the thermophoretic force acting on free optically trapped Janus particles, $F_{t h}^{\text {free }}$, is given by

$$
\left|F_{\text {th }}^{\text {free }}\right|=\left|F_{o p}^{\text {free }}\right|=\left|f k_{r}^{\text {free }} \Delta h_{\text {free }}\right|
$$

The thermophoretic force is only determined by the self-generated temperature gradient around the Janus particle, which is proportional to the beam intensity (Fig. S4), and the nature and concentration of the ions in the medium, defined by the buffer. ${ }^{10}$ To rigorously convert the thermophoretic force between the free and the DNA-tethered, $F_{t h}^{D N A}$, Janus particle we must account for the height differences between the two systems at a given laser power, such that,

$$
F_{t h}^{D N A}=F_{t h}^{f r e e} \cdot \frac{z_{0}^{2}+\Delta h_{\text {free }}^{2}}{z_{0}^{2}+\Delta h_{D N A}^{2}}
$$


(see S7 and S8). Figure 3b exhibits the non-linear relationship between $F_{t h}^{D N A}$ and the laser power, which can be understood considering that the laser intensity varies with both the laser power and the axial position of the particle.

Force-extension data for double-stranded DNA are well-described by the wormlike chain (WLC) model (Figure 4a, solid black line). ${ }^{36}$ However, from the data displayed in Figure $4 \mathrm{a}$ it is clear that the WLC model for dsDNA fails to match our experimental results. The singlemolecule manipulation approach provided by our unique configuration combines force and local thermal gradients. The local temperature in the surface of the gold-coated hemisphere of the Janus particle might affect the double-stranded DNA stability. Hence, we attribute the deviations from the dsDNA WLC behavior to local melting of the DNA molecule: for low laser powers, corresponding to low extensions, low forces, and low temperatures most of the DNA is double-; however as the laser power of the trapping beam increases, the force, the extension and the thermal gradient increases and the proportion of the DNA that is single-stranded is also higher. Double-stranded DNA is considerably stiffer than single-stranded DNA; their respective persistence lengths are $53 \mathrm{~nm}$ for dsDNA and $1.4 \mathrm{~nm}$ for $\operatorname{ssDNA}^{37-40}$ (Figure $4 \mathrm{~b}$ ). The difference in persistence length of double and single-stranded DNA results in pronounced difference of the force-extension response (Figure 4a, solid black and dashed grey lines, respectively). The experimental force-extension curve lies between the curves of ds- and ssDNA, which is consistent with a model that assumes a partial melting of the DNA molecule. Figure 4a also displays the WLC curves for partial DNA dehybridization: $80 \%$ (dotted blue line); $60 \%$ (dotted red line) and $40 \%$ (dotted green line) of dsDNA (see S9). ${ }^{41}$ The close agreement between the experimental results and the WLC curve for a 80 and $60 \%$ dsDNA, supports the hypothesis of having a partially dehybridized DNA tether. However, is important to note that in our 
experimental conditions the proportion of ssDNA to dsDNA is expected to be changed over the course of the force extension curve, since the amount of melted dsDNA depends on the temperature profile, which is set by the laser power and the height of the particle in the optical trap.

DNA in this shear geometry withstands forces $>50 \mathrm{pN},{ }^{42,43}$ well above the forces applied in this study, making it improbable that DNA undergoes force induced melting. Our data strongly suggests that thermal DNA melting occurs in the vicinity of the Janus particle. To quantitatively analyze the effects of the local temperature on the DNA stretching behavior we estimate the temperature difference between the gold and silica hemispheres of the Janus particle as a function of the laser power using the thermophoretic formalism. The thermophoretic force can be described by the Soret coefficient, $S_{\mathrm{T}}$, and it is proportional to the temperature gradient, $\nabla T, 44$

$$
F_{t h}=-S_{T} k_{B} T \nabla T
$$

Following the derivation presented by Sano et al., ${ }^{45}$ the temperature gradient can be expressed as a function of the temperature difference across the two hemispheres of the Janus particles, $\Delta T$. On the other hand, the temperature on the surface of the gold hemisphere is proportional to the light absorbed by the gold layer and it is calculated according to heat transfer equations, such that the thermophoretic force can be described by

$$
F_{t h}=\frac{k_{B} T S_{T} \Delta T}{3 R}
$$

where $\mathrm{R}$ is the radius of the particle (see S7). The Soret coefficient may take positive or negative values depending on the sense of migration of the particles, either from hot to cold $\left(S_{\mathrm{T}}>0\right)$ or 
from cold to hot $\left(S_{\mathrm{T}}<0\right)$. In general, most of the measured Soret coefficient for micro-sized colloidal particles range from $S_{\mathrm{T}}=0.03$ to $0.2 \mathrm{~K}^{-1} \cdot{ }^{44}$ However, experimental values for the Soret coefficient of Janus particles are rare. Experimental conditions and Janus particles similar to our experiments were used by Sano et al., ${ }^{45}$ who studied the active motion of Au-silica Janus particles in a defocused laser beam. They experimentally measured the Soret coefficient of $1 \mu \mathrm{m}$ Au-silica Janus particles using two independent methods and reported values of $10 \mathrm{~K}^{-1}$ and $26 \mathrm{~K}^{-}$ ${ }^{1}$, respectively. ${ }^{45}$ Using Equation (8), the range of $S_{\mathrm{T}}$ values reported by Sano et al. and our experimentally measured thermophoretic forces (Figure 3b), we estimate that the temperature increase around the gold hemisphere of the Janus particles ranges from $3 \pm 1 \mathrm{~K}$ to $230 \pm 102 \mathrm{~K}$, for $F_{\text {th }}$ from 0.1 to $7 \mathrm{pN}$, respectively. To more directly probe the thermal gradient around the Janus particle, we used red fluorescent protein, a thermo sensitive dye, for in situ measurements of the temperature profile. ${ }^{46,47} \mathrm{We}$ found that the fluorescence signal around the gold hemisphere is $\sim 15 \pm 3 \%$ lower than the fluorescence signal around the silica hemisphere, for a trapping laser power of $3.5 \mathrm{~mW}$ (see S10). This fluorescence decrease corresponds to a temperature increase of $8 \pm 4 \mathrm{~K}$, which is consistent, within experimental errors, with the $3 \pm 1 \mathrm{~K}$ temperature increase estimated using the thermophoretic formalism for the same laser power. ${ }^{47}$

To roughly estimate the amount of DNA that separates into single- and double-stranded domains, we model the DNA molecule as a 1D object that is continuously supplied with thermal energy at its thiol end (equating the temperature at the thiol to the surface temperature of the gold hemisphere). We also assume that the temperature around the silica hemisphere of the particle is equal to the ambient temperature. The melting temperature of the 9-kb-long dsDNA used in this study is $59.5 \pm 1.5^{\circ} \mathrm{C}$ at $7.5 \mathrm{mM} \mathrm{Na}^{+}$concentration (see Fig. S6). We found that for an intermediate laser power of $8 \mathrm{~mW}\left(F_{\mathrm{th}}=2.4 \mathrm{pN}\right)$, approximately $80 \pm 12 \%$ of the total length of 
the DNA should remain double-stranded and the remaining $20 \pm 12 \%$ is single-stranded. The error bars correspond to the different Soret coefficient of $10 \mathrm{~K}^{-1}$ and $26 \mathrm{~K}^{-1}$. Even though this approximation is very rough (i.e: we assume that heat dissipation towards the silica hemisphere is negligible and that heat dissipation from the gold to the DNA is a 1D process) the estimated percentages of dsDNA and ssDNA correspond fairly well with the experimental force-extension curve which lies between the theoretical WLC curve for a 80 and $60 \%$ dsDNA to ssDNA ratio.

The DNA tether ruptures at trapping laser powers between 9 and $11 \mathrm{~mW}$ (Fig. S3), which corresponds to forces of 4 to $7 \mathrm{pN}$. Our attachment geometry, with a single thiol and single digoxigenin linkage on opposite strands, implies that complete DNA melting would result in loss of the tether. However, the temperature at the gold surface required to completely thermally separate both strands of DNA, modeled as a 1D object, is well above the maximum thermal increase estimated in our experiments ( $75 \mathrm{~K}$ above the ambient at the Janus particle surface). A likely pathway for unbinding of the tether is through loss of the digoxigenin-antidigoxigenin surface attachment. The DIG-antiDIG bond has been investigated in a number of force spectroscopy experiments ${ }^{29,48}$. While there is a considerable spread in the literature values for the force-dependent off-rates of the DIG-antiDIG bond, the results suggest that even the relatively moderate forces applied in our experiments would reduce the lifetime to $\sim 1-10 \mathrm{~min}$, which is the approximate time scale of our experiments, 5-7 min per particle (see S12). Regarding the Au-S covalent bond, the rupture force of this single bond is in the order of $\sim 1 \mathrm{nN},{ }^{49}$ well above the forces achieved with our configuration. However, experiments demonstrate that the thiol-Au bond is susceptible to thermal rupture at temperatures above $80-90^{\circ} \mathrm{C} .{ }^{50-52}$ Therefore, it is likely that both the force-induced dissociation of the DIG-antiDIG bond and the thermally induced 
breakage of the thiol bond to the gold hemisphere of the Janus particle contribute to the observed tether rupture.

\section{Conclusions}

In summary, we demonstrate that optically trapped Janus particles can stretch single dsDNA molecules tethered between a micron-size particle and a glass substrate. We take advantage of the light induced thermophoretic force to control the extension of the DNA molecule. Further, the temperature increase around the gold hemisphere of the particle provides an efficient approach to simultaneously heat and stretch the dsDNA molecule. By combining optical and thermophoretic forces, we can achieve complete extension of the DNA tether with forces ranging between 0.1 and $7 \mathrm{pN}$ and we can simultaneously partially melt it. Our experiments demonstrate that introducing thermophoretic effects adds new capabilities to the widely used conventional optical tweezers configuration. While the rotational orientation (and therefore propulsion direction) of the Janus particle is determined by the direction of the external optical field, the stretching capabilities are driven by the thermophoretic forces generated by the asymmetrical nature of the particle.

We anticipate that our approach can be extended further in a number of exciting directions, in particular by decoupling stretching from heating. For example, by carefully engineering plasmonics Janus particles using different sizes of silica spheres and/or different thicknesses and treatments of the gold coating, it is possible to make accessible specific force and temperature ranges. Furthermore, designing particles using several different coatings and 'patches' could confer custom-engineered optical and thermal properties. ${ }^{53-56}$ 
In addition to engineering Janus particles, the laser system can be optimized to control the interplay of optical and thermophoretic forces,${ }^{57}$ e.g. by shaping the laser beam, by using several lasers at different wavelengths, or adding electrical fields. ${ }^{58,59}$ The flexibility of this approach could open up possibilities to study e.g. the interplay of force and thermal stability for both nucleic acids and proteins, complementary to other methods. ${ }^{60-62}$ In particular the ability to generate heat locally, in the vicinity of the trapped particle, opens up possibilities for fast switching and design of the temperature profiles. ${ }^{63,64,25}$

\section{Methods}

Au-silica Janus particles synthesis and characterization. Au-silica Janus particles were synthesized following a procedure described elsewhere. ${ }^{9}$ Briefly, $1.3 \mu \mathrm{m}$ silica spheres (Microparticles $\mathrm{GmbH}$ ) were centrifuged and redispersed in a $0.5 \% \mathrm{w} / \mathrm{w}$ hexylamine ethanolic solution.Then, the colloidal suspension was self-assembled onto a plasma cleaned glass coverslips. The monolayer of silica spheres was prepared on a water surface by slowly pumping out the excess of solvent. Subsequently, the substrates were placed in a thermal evaporator where a layer of gold ( $\sim 6 \mathrm{~nm}$ thickness) was deposited at a rate of $0.1 \mathrm{~nm}$ per second. Finally, the particles were suspended in miliQ water by sonicating the glass substrate in a water filled beaker. Au-silica Janus particles were imaged by scanning electron microscopy (SEM) in a Gemini Ultra Plus equipment with a nominal resolution of $\sim 2 \mathrm{~nm}$ (Zeiss). White light scattering spectra of individual Au-silica Janus particles in water were acquired in an Acton SP2500 spectrometer (Princeton Instruments) coupled to an upright Zeiss Axio Scope A1 (Zeiss) microscope equipped with 100x, 1.0 NA water immersion Zeiss Achroplan objective (Zeiss) and an oil immersion dark-field condenser (NA = 1.2-1.4). A $100 \mathrm{~W}$ halogen lamp (Zeiss) was used for illumination. 
DNA synthesis and characterization. In order to avoid approaching the secondary optical trap, positioned a few micrometer above the focus of the laser beam, we designed a double-stranded DNA molecule of 9046 basepairs, which corresponds to a contour length of approx. $3 \mu \mathrm{m}$. For the polymerase chain reaction (PCR) we used $\lambda$-DNA (Fermentas) as template $(15 \mathrm{mg} / \mathrm{L})$, a forward primer functionalized with a thiol group and a reverse primer functionalized with a digoxigenin label at the $5^{\prime}$ end (each at $0.5 \mu \mathrm{M}$ ). We designed the primers using an online tool called the OligoAnalyzer from idtdna.com and ordered the sequences at Biomers.net. For the PCR reaction we used a commercial available Phusion Mix (Thermo Scientific). We used a ProFlex PCR System for the temperature cycling and added $3 \%$ DMSO to the solution to increase yield. The DNA was purified using a ready-to-use kit by Qiagen (QIAquick PCR Purification Kit). The final yield was $55 \mu \mathrm{L}$ of DNA at $28.5 \mathrm{mg} / \mathrm{L}$, determined by UV-Vis (Thermo Scientific) absorption. The reaction product was visualized on a $1 \%$ agarose gel (High resolution Agarose, Sigma-Aldrich) and compared to a DNA ladder (1 kbp DNA ladder, NEB). Functionalization of Au-silica Janus particles with DNA. To perform the optical trapping experiments solutions of Au-silica Janus particles with and without DNA were prepared by diluting the stock colloidal dispersion of Janus particles in $5 \mathrm{mM}$ phosphate buffer, $\mathrm{pH}$ 7.2, supplemented with $7.5 \mathrm{Mm} \mathrm{NaCl}$ buffer (without potasium) and allowing it to mix overnight at 4 ${ }^{\circ} \mathrm{C}$. The concentration of Janus particles stock solution was estimated to be $\sim 50 \mathrm{fM}$. The concentration of Au-silica Janus particles, DNA, sodium phosphate buffer (Thermo Scientific) and $\mathrm{NaCl}$ in the final solution was $12 \mathrm{fM}, 120 \mathrm{pM}, 5 \mathrm{mM}$ and $7.5 \mathrm{mM}$, respectively. Au-silica Janus without DNA (free Janus particles) were prepared with the same particle and buffer concentration as described above, but replacing the amount of DNA solution with buffer. The 
ratio of Janus particles to DNA was experimentally optimized by testing different reactions conditions as detailed in S2.

Attachment of DNA-functionalized Au-silica Janus particles to coverslides. To fix the DNA molecule to the surface, i.e. the glass coverslip, we first coat the glass with epoxy silan, as commonly used in single-molecule experiments. Briefly, glass coverslips freshly cleaned in piranha solution are gently stirred for $1 \mathrm{hs}$ and in the dark in a $88 \%$ ethanol and a $2 \% 3$ Aminopropyldimethylethoxysilane aqueous solution. The epoxy silan functionalized cover slides are rinsed with ethanol and water, placed in an $80{ }^{\circ} \mathrm{C}$ oven for $30 \mathrm{~min}$ and stored under $\mathrm{N}_{2}$ for up to 6 months. We directly place a droplet of anti-dixoxigenin $(40 \mu \mathrm{L}$ at $100 \mathrm{mg} / \mathrm{mL})$ on the glass coverslip and let it incubate for at least $10 \mathrm{~min}$. The substrate was then thoroughly cleaned, four times, using phosphate buffer (same concentration as described above). For passivation we placed a droplet of Tween $(60 \mu \mathrm{L}$ at $0.05 \%$ in $20 \mathrm{mM}$ TRIS-HCl and $5 \mathrm{Mm}$ EDTA, pH 7.6) onto the glass cover slip (incubation time $\sim 5 \mathrm{~min}$.). Next, the substrate was cleaned again using four times phosphate buffer. Finally, we placed the DNA/Janus solution (as described above) onto the glass coverslip and directly started the measurements.

Microelevation microscopy experiments and data analysis. Free and DNA-tethered Au-silica Janus particles microelevation experiments were performed in an upright Zeiss Axio Scope A1 (Zeiss) microscope in conjunction with an oil immersion dark-field condenser (NA = 1.2-1.4). The optical trap was formed by tightly focusing a $1064 \mathrm{~nm}$ Cobolt Rumba ${ }^{\mathrm{TM}} \mathrm{CW}$ laser beam (Cobolt AB) with a 100x, 1.0 NA water immersion Zeiss Achroplan objective (Zeiss). The final power of the laser beam at the objective was optimized using neutral density filters between 1 to $20 \mathrm{~mW}$. Dark field imaging videos were collected with a digital SLR Canon EOS 550D camera (Canon) with a resolution of $1920 \times 1080$ operated at a frame rate of $29.9 \mathrm{fps}$ using the same 
objective. Typical microelevation experiments were performed by depositing a $80 \mu \mathrm{L}$ drop of the corresponding solution (free or DNA-functionalized Au-silica Janus particles, in $5 \mathrm{mM}$ phosphate buffer, $\mathrm{pH} 7.2$, with $7.5 \mathrm{Mm} \mathrm{NaCl}$ ) either over an DIG-antiDIG functionalized microscope coverslips (Menzel-Glaser \# 1.5) or over a cleaned glass substrate (control experiments). Glass coverslips were cleaned by sonication for $20 \mathrm{~min}$ in each of the following solvents: 2-isopropanol, actone, miliQ water and then dried with $\mathrm{N}_{2}$. The height of the Janus particle in the optical trap was determined by analysis of the field distribution pattern of the recorded dark-field videos. The calibration between the diffraction pattern of the white light scattering of the particle and the height was performed using a reference sample of Au-silica Janus particles fixed over a coverslip, which were axially displaced with respect to the microscope objective using a PI 3D piezo driven nanopositioner stage (Physik Instrumente $\mathrm{GmbH})$. The data analysis was performed using a homemade MATLAB routine that tracks the height and the $x, y$ positions of the particle during the time of the experiment.

Temperature measurments. To measure in-situ the temperature increase around the optically trapped Au-silica Janus particles, red fluorescent protein was added to the free Janus particles solution at a final concentration of $1.5 \mu \mathrm{M}$. Red fluorescent protein was excited with a OPUS $\mathrm{CW}$ laser $532 \mathrm{~nm}$ laser (Laser Quantum) coupled and collimated into the microscope. The fluorescent signal was passed through a $532 \mathrm{~nm}$ notch filter (Thorlabs) and collected with an iXon Ultra 897 EM-CCD (Andor) with $512 \times 512$ pixels of $16 \mu \mathrm{m} \times 16 \mu \mathrm{m}$ size, operated at $100 \mathrm{~ms}$ per frame. The fluorescence of the red fluorescence protein was measured while simultaneously trapping the Au-silica Janus particle with the $1064 \mathrm{~nm}$ laser beam. To avoid photobleaching of the fluorophore, the white light source used in dark field microscopy was immediately turned off after optically trapping the Janus particle. The $532 \mathrm{~nm}$ laser was then turn 
on to collect the fluorescence signal of the protein. Fluorescence intensity analysis was performed with a homemade MATLAB routine. To calculate the decrease in fluorescence signal around the gold-coated hemisphere of the Janus particle, we integrated the fluorescence intensity at each side of the Janus particle and fitted each side of the data with a Gaussian function of equal width.

dsDNA Melting Temperature Determination. We used a thermo-cycler and fluorescence read out to determine the melting temperature of the 9-kbp-DNA construct used in the Janus particlebased stretching experiments. For the fluorescence read out, we mixed DNA (final concentration $11 \mathrm{pM}$ ) with the fluorescent dye EvaGreen at 1x final concentration according to the vendor's specification (Biotium) in the same buffer that was used for single-molecule experiments (20x diluted phosphate buffer saline, PBS, ThermoFisher). We ran a temperature gradient from $95{ }^{\circ} \mathrm{C}$ to $5{ }^{\circ} \mathrm{C}$ (and back) in steps of $1{ }^{\circ} \mathrm{C}$ every minute. The melting temperature $\mathrm{T}_{\mathrm{m}}$ was determined similarly to reference 65 by performing a baseline fit and defining the median. ${ }^{65}$ From the fit, $\mathrm{T}_{\mathrm{m}}$ was determined, i.e. the temperature at which half of DNA bases are single-stranded and half are double-stranded, see Supplementary Figure S6. For comparison, we also determined the $\mathrm{T}_{\mathrm{m}}$ at a higher buffer concentration (1x diluted phosphate buffer saline, PBS, ThermoFisher).

FDTD Simulations. FDTD simulations of Au-silica Janus particles (particle diameter $1.3 \mu \mathrm{m}$, thickness of half-hemisphere gold cap $5 \mathrm{mn}$ ) were carried out using Lumerical FDTD solutions (Lumerical Solutions, Canada). Optical constant data tables were taken from Johnson and Christie for gold, and Palik for the dielectric surrounding (water as effective medium).

\section{Associated Content}

Supporting Information: This material is available free of charge on the ACS Publications website at http://pubs.acs.org. 
Optical characterization of Au-silica Janus particles, tethering efficiency of single DNA molecules to Au-silica Janus particles, forces acting on a free optically trapped Au-silica Janus particle, axial displacement of optically trapped DNA-tethered Au-silica Janus particles, axial optical forces in the limit of small radial displacements, thermophoretic force on free Au-silica Janus particles, free to DNA-tethered thermophoretic force conversion, force-extension curve of a partially de-hybridized DNA molecule, temperature distribution around an optically trapped Janus particle, melting curve of the 9-kb-long dsDNA and lifetime of DIG-antiDIG bond.

\section{Author Information}

Corresponding Authors:

*E-mail: s.simoncelli@imperial.ac.uk

*E-mail: Jan.Lipfert@lmu.de

Notes: The authors declare no competing financial interest.

\section{Acknowledgements}

This work has been supported by the ERC through the Advanced Investigator Grant "HYMEM", the German Research Foundation (DFG) through Sonderforschungsbereich SFB863, and a Fulbright fellowship to S.J.

\section{References}

(1) Wong, F.; Dey, K. K.; Sen, A. Synthetic Micro/Nanomotors and Pumps: Fabrication and Applications. Annu. Rev. Mater. Res. 2016, 46, 407-432.

(2) Abdelmohsen, L. K. E. A.; Peng, F.; Tu, Y.; Wilson, D. A. Micro- and Nano-Motors for Biomedical Applications. J. Mater. Chem. B 2014, 2, 2395-2408. 
(3) Gao, W.; Wang, J. Synthetic Micro/nanomotors in Drug Delivery. Nanoscale 2014, 6, 10486-10494.

(4) Xi, W.; Solovev, A. a.; Ananth, A. N.; Gracias, D. H.; Sanchez, S.; Schmidt, O. G. Rolledup Magnetic Microdrillers: Towards Remotely Controlled Minimally Invasive Surgery. Nanoscale 2013, 5, 1294-1297.

(5) Wu, Z.; Lin, X.; Wu, Y.; Si, T.; Sun, J.; He, Q. Near-Infrared Light-Triggered On/off Motion of Polymer Multilayer Rockets. ACS Nano 2014, 8, 6097-6105.

(6) Buttinoni, I.; Volpe, G.; Kümmel, F.; Volpe, G.; Bechinger, C. Active Brownian Motion Tunable by Light. J. Phys. Condens. Matter 2012, 24, 284129.

(7) Ilic, O.; Kaminer, I.; Lahini, Y.; Buljan, H.; Soljačić, M. Exploiting Optical Asymmetry for Controlled Guiding of Particles with Light. ACS Photonics 2016, 3, 197-202.

(8) Moyses, H.; Palacci, J.; Sacanna, S.; Grier, D. G. Trochoidal Trajectories of SelfPropelled Janus Particles in a Diverging Laser Beam. Soft Matter 2016, 12, 6357-6364.

(9) Nedev, S.; Carretero-Palacios, S.; Kühler, P.; Lohmüller, T.; Urban, A. S.; Anderson, L. J. E.; Feldmann, J. An Optically Controlled Microscale Elevator Using Plasmonic Janus Particles. ACS photonics 2015, 2, 491-496.

(10) Simoncelli, S.; Summer, J.; Nedev, S.; Kühler, P.; Feldmann, J. Combined Optical and Chemical Control of a Microsized Photofueled Janus Particle. Small 2016, 12, 2854-2858.

(11) Ashkin, A.; Dziedzic, J. M.; Bjorkholm, J. E.; Chu, S. Observation of a Single-Beam Gradient Force Optical Trap for Dielectric Particles. Opt. Lett. 1986, 11, 288.

(12) Lehmuskero, A.; Johansson, P.; Rubinsztein-Dunlop, H.; Tong, L.; Käll, M. Laser Trapping of Colloidal Metal Nanoparticles. ACS Nano 2015, 9, 3453-3469.

(13) Maragò, O. M.; Jones, P. H.; Gucciardi, P. G.; Volpe, G.; Ferrari, A. C. Optical Trapping 
and Manipulation of Nanostructures. Nat. Nanotechnol. 2013, 8, 807-819.

(14) Oddershede, L. B.; Bahadori, A.; Bendix, P. M. Optical Manipulation of Hot Nanoparticles Can Mediate Selected Cell Fusion. In; 2017; Vol. 10252, pp. $1025203-$ 1025207.

(15) Rørvig-Lund, A.; Bahadori, A.; Semsey, S.; Bendix, P. M.; Oddershede, L. B. Vesicle Fusion Triggered by Optically Heated Gold Nanoparticles. Nano Lett. 2015, 15, 41834188.

(16) Ashkin, A.; Dziedzic, J. M.; Yamane, T. Optical Trapping and Manipulation of Single Cells Using Infrared Laser Beams. Nature 1987, 330, 769-771.

(17) Bustamante, C.; Bryant, Z.; Smith, S. B. Ten Years of Tension: Single-Molecule DNA Mechanics. Nature 2003, 421, 423-427.

(18) Allemand, J. F.; Bensimon, D.; Croquette, V. Stretching DNA and RNA to Probe Their Interactions with Proteins. Current Opinion in Structural Biology, 2003, 13, 266-274.

(19) Neuman, K. C.; Nagy, A. Single-Molecule Force Spectroscopy: Optical Tweezers, Magnetic Tweezers and Atomic Force Microscopy. Nat. Methods 2008, 5, 491-505.

(20) Puchner, E. M.; Gaub, H. E. Single-Molecule Mechanoenzymatics. Annu. Rev. Biophys. 2012, 41, 497-518.

(21) Neuman, K. C.; Abbondanzieri, E. A.; Landick, R.; Gelles, J.; Block, S. M. Ubiquitous Transcriptional Pausing Is Independent of RNA Polymerase Backtracking. Cell, 2003, $115,437-447$.

(22) Abbondanzieri, E. A.; Greenleaf, W. J.; Shaevitz, J. W.; Landick, R.; Block, S. M. Direct Observation of Base-Pair Stepping by RNA Polymerase. Nature 2005, 438, 460-465.

(23) Inman, J.; Forth, S.; Wang, M. D. Passive Torque Wrench and Angular Position Detection 
Using a Single-Beam Optical Trap. Opt. Lett. 2010, 35, 2949-2951.

(24) Wang, L.; Xu, X.; Kumar, R.; Maiti, B.; Liu, C. T.; Ivanov, I.; Lee, T. H.; Benkovic, S. J. Probing DNA Clamps with Single-Molecule Force Spectroscopy. Nucleic Acids Res. 2013, 41, 7804-7814.

(25) Osinkina, L.; Carretero-Palacios, S.; Stehr, J.; Lutich, A. A.; Jäckel, F.; Feldmann, J. Tuning DNA Binding Kinetics in an Optical Trap by Plasmonic Nanoparticle Heating. Nano Lett. 2013, 13, 3140-3144.

(26) Meglio, A.; Praly, E.; Ding, F.; Allemand, J. F.; Bensimon, D.; Croquette, V. Single DNA/protein Studies with Magnetic Traps. Current Opinion in Structural Biology, 2009, $19,615-622$.

(27) Dulin, D.; Lipfert, J.; Moolman, M. C.; Dekker, N. H. Studying Genomic Processes at the Single-Molecule Level: Introducing the Tools and Applications. Nat. Rev. Genet. 2012, $14,9-22$.

(28) Yang, D.; Ward, A.; Halvorsen, K.; Wong, W. P. Multiplexed Single-Molecule Force Spectroscopy Using a Centrifuge. Nat. Commun. 2016, 7, 11026.

(29) Sitters, G.; Kamsma, D.; Thalhammer, G.; Ritsch-Marte, M.; Peterman, E. J. G.; Wuite, G. J. L. Acoustic Force Spectroscopy. Nat. Methods 2015, 12, 47-50.

(30) Sitters, G.; Laurens, N.; de Rijk, E.; Kress, H.; Peterman, E. J. G.; Wuite, G. J. L. Optical Pushing: A Tool for Parallelized Biomolecule Manipulation. Biophys. J. 2017, 110, 4450.

(31) Shon, M. J.; Cohen, A. E. Nano-Mechanical Measurements of Protein-DNA Interactions with a Silicon Nitride Pulley. Nucleic Acids Res. 2016, 44, e7.

(32) Bustamante, C.; Marko, J. F.; Siggia, E. D.; Smith, S. Entropic Elasticity of Lambda- 
Phage DNA. Science 1994, 265, 1599-1600.

(33) Peterman, E. J. G.; Gittes, F.; Schmidt, C. F. Laser-Induced Heating in Optical Traps. Biophys. J. 2003, 84, 1308-1316.

(34) Smith, S.; Finzi, L.; Bustamante, C. Direct Mechanical Measurements of the Elasticity of Single DNA Molecules by Using Magnetic Beads. Science. 1992, 258, 1122-1126.

(35) Yehoshua, S.; Pollari, R.; Milstein, J. N. Axial Optical Traps: A New Direction for Optical Tweezers. Biophysical Journal, 2015, 108, 2759-2766.

(36) Marko, J. F.; Siggia, E. D. Stretching DNA. Macromolecules 1995, 28, 8759-8770.

(37) Bosco, A.; Camunas-Soler, J.; Ritort, F. Elastic Properties and Secondary Structure Formation of Single-Stranded DNA at Monovalent and Divalent Salt Conditions. Nucleic Acids Res. 2014, 42, 2064-2074.

(38) Sim, A. Y. L.; Lipfert, J.; Herschlag, D.; Doniach, S. Salt Dependence of the Radius of Gyration and Flexibility of Single-Stranded DNA in Solution Probed by Small-Angle XRay Scattering. Phys. Rev. E 2012, 86, 21901.

(39) Wenner, J. R.; Williams, M. C.; Rouzina, I.; Bloomfield, V. A. Salt Dependence of the Elasticity and Overstretching Transition of Single DNA Molecules. Biophys. J. 2002, 82, $3160-3169$.

(40) Baumann, C. G.; Smith, S. B.; Bloomfield, V. a; Bustamante, C. Ionic Effects on the Elasticity of Single DNA Molecules. Proc. Natl. Acad. Sci. U. S. A. 1997, 94, 6185-6190.

(41) Suksombat, S.; Khafizov, R.; Kozlov, A. G.; Lohman, T. M.; Chemla, Y. R. Structural Dynamics of E. Coli Single-Stranded DNA Binding Protein Reveal DNA Wrapping and Unwrapping Pathways. Elife 2015, 4, e08193.

(42) Kufer, S. K.; Puchner, E. M.; Gumpp, H.; Liedl, T.; Gaub, H. E. Single-Molecule Cut- 
and-Paste Surface Assembly. Science. 2008, 319, 594.

(43) Smith, S. B.; Cui, Y.; Bustamante, C. Overstretching B-DNA: The Elastic Response of Individual Double-Stranded and Single-Stranded DNA Molecules. Science. 1996, 271, 795-799.

(44) Würger, A. Thermal Non-Equilibrium Transport in Colloids. Reports Prog. Phys. 2010, $73,126601$.

(45) Jiang, H. R.; Yoshinaga, N.; Sano, M. Active Motion of a Janus Particle by SelfThermophoresis in a Defocused Laser Beam. Phys. Rev. Lett. 2010, 105, 268302.

(46) Deepankumar, K.; Nadarajan, S. P.; Bae, D.-H.; Baek, K.-H.; Choi, K.-Y.; Yun, H. Temperature Sensing Using Red Fluorescent Protein. Biotechnol. Bioprocess Eng. 2015, $20,67-72$.

(47) Ishii, M.; Kunimura, J. S.; Penna, T. C. V.; Cholewa, O. Study on the Thermal Stability of Green Fluorescent Protein (GFP) in Glucose Parenteral Formulations. Int. J. Pharm. 2007, 337, 109-117.

(48) Neuert, G.; Albrecht, C.; Pamir, E.; Gaub, H. E. Dynamic Force Spectroscopy of the Digoxigenin-Antibody Complex. FEBS Lett. 2006, 580, 505-509.

(49) Grandbois, M.; Beyer, M.; Rief, M.; Clausen-Schaumann, H.; Gaub, H. E. How Strong Is a Covalent Bond? Science. 1999, 283, 1727-1730.

(50) Gustafson, T. P.; Cao, Q.; Wang, S. T.; Berezin, M. Y. Design of Irreversible Optical Nanothermometers for Thermal Ablations. Chem. Commun. 2013, 49, 680-682.

(51) Borzenkov, M.; Chirico, G.; D’Alfonso, L.; Sironi, L.; Collini, M.; Cabrini, E.; Dacarro, G.; Milanese, C.; Pallavicini, P.; Taglietti, A.; et al. Thermal and Chemical Stability of Thiol Bonding on Gold Nanostars. Langmuir 2015, 31, 8081-8091. 
(52) Goodman, A. M.; Hogan, N. J.; Gottheim, S.; Li, C.; Clare, S. E.; Halas, N. J.

Understanding Resonant Light-Triggered DNA Release from Plasmonic Nanoparticles. ACS Nano 2017, 11, 171-179.

(53) Spesyvtseva, S. E. S.; Dholakia, K. Trapping in a Material World. ACS Photonics 2016, 3, 719-736.

(54) Kamalasanan, K.; Jhunjhunwala, S.; Wu, J.; Swanson, A.; Gao, D.; Little, S. R. Patchy, Anisotropic Microspheres with Soft Protein Islets. Angew. Chemie - Int. Ed. 2011, 50, 8706-8708.

(55) Yi, Y.; Sanchez, L.; Gao, Y.; Yu, Y. Janus Particles for Biological Imaging and Sensing. Analyst 2016, 141, 3526-3539.

(56) Ma, H.; Tian, P.; Pello, J.; Bendix, P. M.; Oddershede, L. B. Heat Generation by Irradiated Complex Composite Nanostructures. Nano Lett. 2014, 14, 612-619.

(57) Bregulla, A. P.; Yang, H.; Cichos, F. Stochastic Localization of Microswimmers by Photon Nudging. ACS Nano 2014, 8, 6542-6550.

(58) Nishiguchi, D.; Sano, M. Mesoscopic Turbulence and Local Order in Janus Particles SelfPropelling under an Ac Electric Field. Phys. Rev. E - Stat. Nonlinear, Soft Matter Phys. 2015, 92 .

(59) Mano, T.; Delfau, J.-B.; Iwasawa, J.; Sano, M. Optimal Run-and-Tumble--based Transportation of a Janus Particle with Active Steering. Proc. Natl. Acad. Sci. 2017, 114, E2580-E2589.

(60) De Lorenzo, S.; Ribezzi-Crivellari, M.; Arias-Gonzalez, J. R.; Smith, S. B.; Ritort, F. A Temperature-Jump Optical Trap for Single-Molecule Manipulation. Biophys. J. 2015, 108, $2854-2864$. 
(61) Schlierf, M.; Rief, M. Temperature Softening of a Protein in Single-Molecule Experiments. J. Mol. Biol. 2005, 354, 497-503.

(62) Dupuis, N. F.; Holmstrom, E. D.; Nesbitt, D. J. Single-Molecule Kinetics Reveal CationPromoted DNA Duplex Formation through Ordering of Single-Stranded Helices. Biophys. J. 2013, 105, 756-766.

(63) Cichos, F.; Thalheim, T.; Mertig, M.; Guenther, K. Manipulating Single and Multiple Biomolecules with Dynamic Temperature Fields. In Optics in the Life Sciences Congress; OSA Technical Digest (online); Optical Society of America: San Diego, California , 2017; p. OtM3E.1.

(64) Stehr, J.; Hrelescu, C.; Sperling, R. A.; Raschke, G.; Wunderlich, M.; Nichtl, A.; Heindl, D.; Kürzinger, K.; Parak, W. J.; Klar, T. A.; et al. Gold NanoStoves for Microsecond DNA Melting Analysis. Nano Lett. 2008, 8, 619-623.

(65) Mergny, J. L.; Lacroix, L. Analysis of Thermal Melting Curves. Oligonucleotides 2003, $13,515-537$. 


\section{Figures}

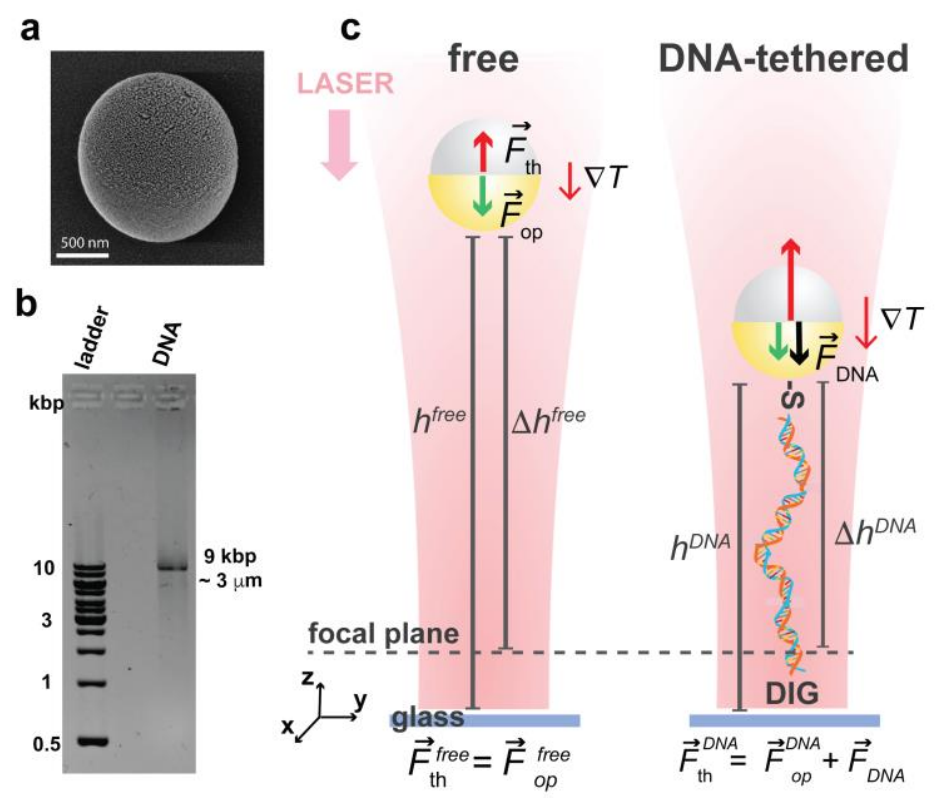

Figure 1. (a) Representative SEM image of a gold-silica Janus particle. The electron micrograph of the Janus particle shows the gold surface roughness. (b) Gel-electrophoresis image of a molecular-weight ladder and the reaction product from the dual functionalized dsDNA PCR reaction. (c) Schematic representation of our thermophoretic-force optical tweezers configuration and force diagram illustrating the axial main forces acting on free and DNA-tethered optically trapped gold-silica Janus particles. A gold-silica Janus particle is either free in solution or tethered to a glass surface by a $9 \mathrm{kbp}$ ( $\sim 3 \mu \mathrm{m}$ contour length) dsDNA molecule and it is optically trapped by a focused laser beam. The DNA tether is attached via digoxigenin and antidigoxigenin interaction on the glass surface, and via a thiol bond to the gold face of the particle. For free Janus particles, the thermophoretic force, $F_{\text {th }}$, balances the optical forces from the laser particle interaction, $F_{\text {op }}$. However, for DNA-tethered particles, the resultant tension force of the tether shifts the particle from its stable trapping position at $h_{\text {free }}$ to $h_{D N A}$. The DNA molecule is thus subject to a restoring force, $F_{\mathrm{DNA}}$, given by the difference between the thermophoretic and optical forces at the DNA-tethered particle height. The gravitational and buoyancy forces are negligible and are therefore not shown in the diagram for clarity. 

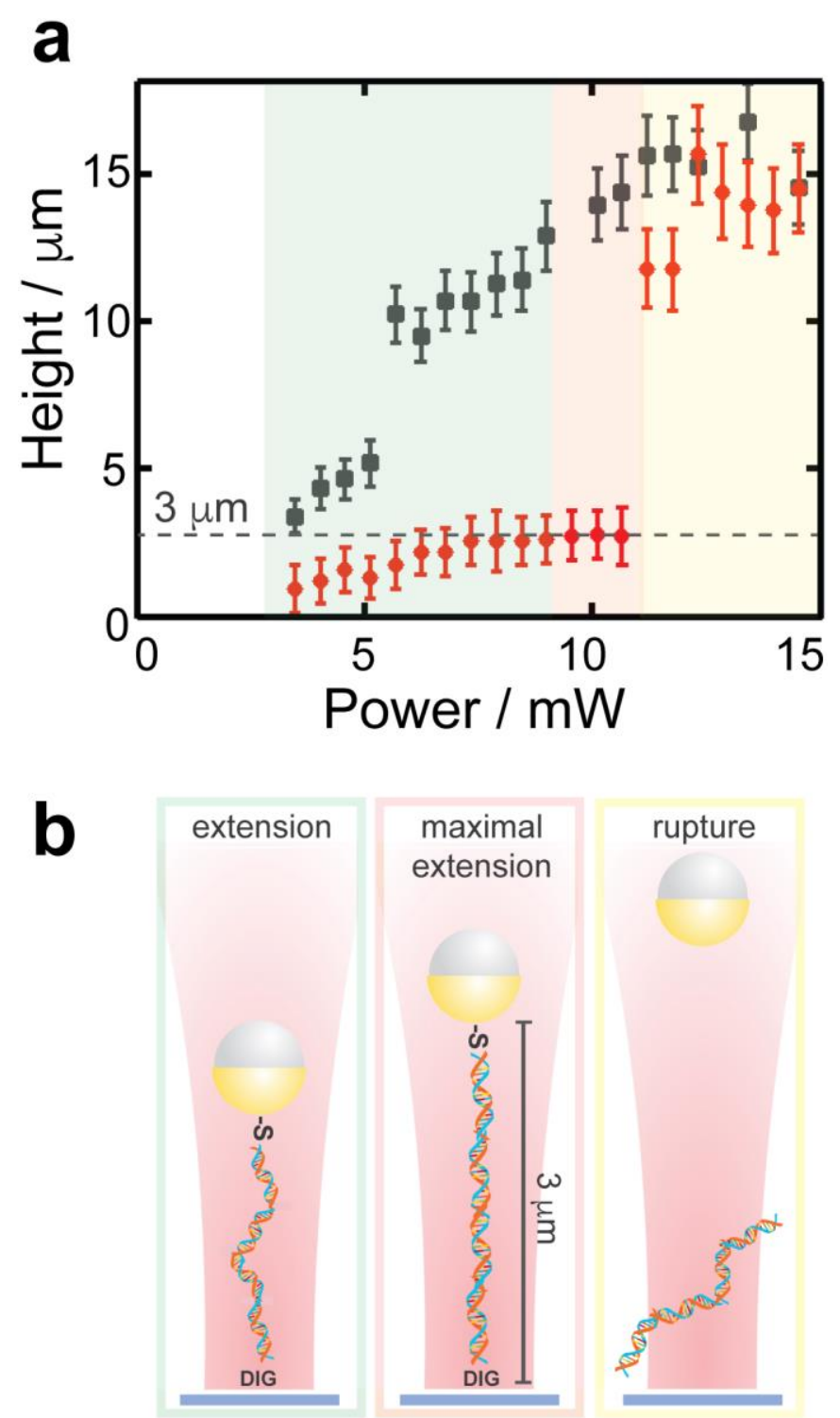

Figure 2. (a) Height of a DNA-tethered (red circles) and a free (grey squares) Au-silica Janus particle as a function of the trapping laser power. The contour length of the DNA construct is 3 $\mu \mathrm{m}$ and indicated by the dashed line. (b) Schematic representation of the three different colorcoded regimes observed in a for the DNA-tethered Au-silica Janus particle experiment. The DNA-tethered particle elevates in the optical trap until reaching a plateau height equal to the DNA contour length (maximal extension). The DNA-tethered ruptures at higher laser powers. 
a

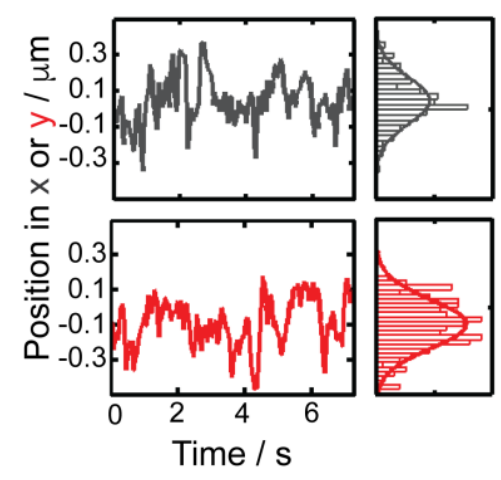

b

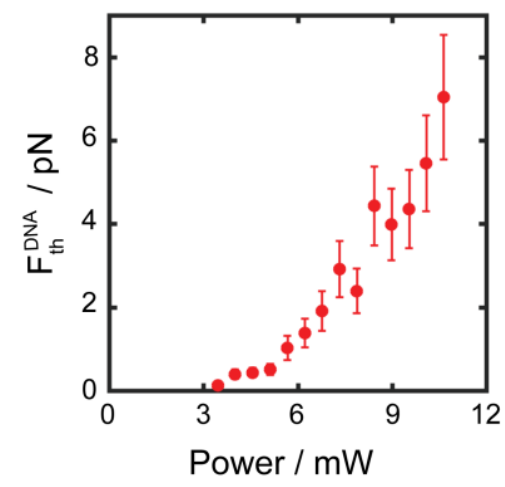

Figure 3. (a) Temporal traces of the $x$ and $y$ position of a free Au-silica Janus particle that is optically trapped at a fixed laser power. (b) Thermophoretic force acting on the DNA-tethered $\mathrm{Au}$-silica Janus particles as a function of the trapping laser power.

a

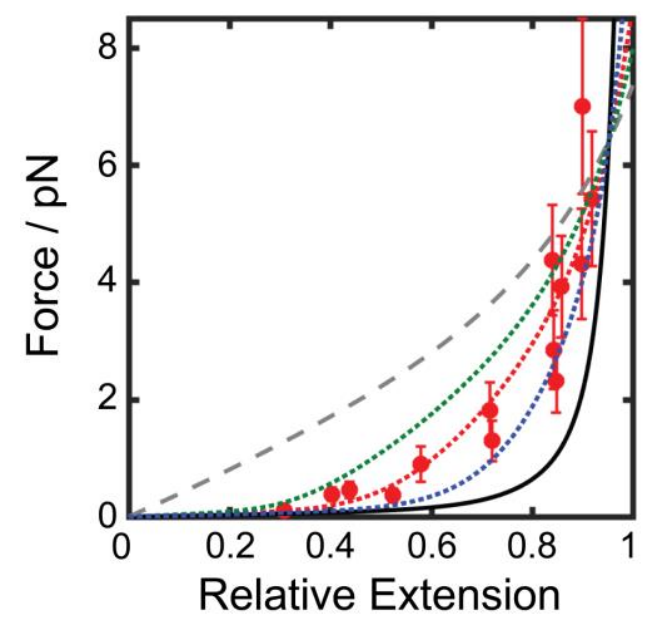

b

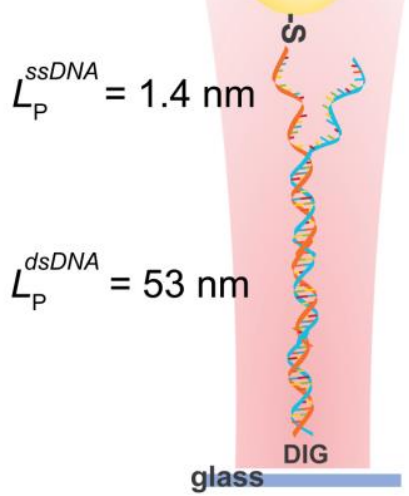

Figure 4. (a) Force extension curve of a DNA molecule tethered between an optically trapped Au-silica Janus particle and a glass surface (red circles). The relative extension was normalized to the contour length of the dsDNA of $3 \mu \mathrm{m}$. The WLC theoretical force-extension curves for a fully dsDNA (solid black line), a fully ssDNA (dashed grey line) and for partially melted ss/dsDNA of $80 \%$ (dotted blue line); $60 \%$ (dotted red line) and $40 \%$ (dotted green line) content of dsDNA at a temperature of $100{ }^{\circ} \mathrm{C}$ are plotted for comparison. (b) Schematic representation of the partial melting model. The tethered DNA molecule is simultaneously subject to force and heat. The increase in temperature around the gold hemisphere of the Janus 
particle partially melts the DNA tether. The persistence length of the ds- and ssDNA are $53 \mathrm{~nm}$ and $1.4 \mathrm{~nm}$, respectively. 


\title{
For Table of Contents Use Only
}

\section{Stretching and heating single DNA molecules with optically trapped gold-}

\section{silica Janus particles}

\author{
Sabrina Simoncelli ${ }^{1 \dagger *}$, Samuel Johnson ${ }^{1 \ddagger}$, Franziska Kriegel ${ }^{2}$, Jan Lipfert ${ }^{2,3 *}$ and Jochen \\ Feldmann $^{1,3}$
}
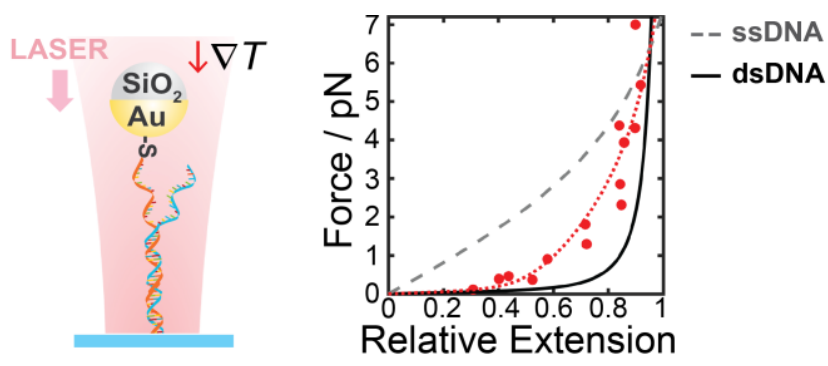

The optical elevation of a micro-scale bifacial gold-silica particle inside an optical trap can be exploited to efficiently heat and stretch single dsDNA molecules. 